\title{
Nervous System
}

8.5 Cytokines, Selectins and Adhesion Molecules Plasma Levels in Acute Ischaemic Stroke: Relationship with Stroke Subtype, Ischaemic Lesion Site and Clinical Outcome

A. Tuttolomondo, A. Pinto, D. Di Raimondo, S. Corrao, R. Di Sciacca, G. Licata

Biomedical Department of Internal and Specialistic Medicine, University of Palermo, Italy

Introduction: No study, to our knowledge, has evaluated immunoinflammatory activation of the acute phase in relation to each diagnostic subtype of ischaemic stroke and few studies examined the relationship between inflammatory biomarkers blood levels and stroke outcome. On this basis the aim of our study is to evaluate in patients with acute ischaemic stroke cytokines, selectins and adhesion molecules plasma levels in relation to diagnostic subtype and to evaluate the relationship between immunoinflammatory variables and clinical outcome.

Methods: We enrolled all consecutive patients with a diagnosis of acute ischaemic stroke and control patients without a diagnosis of acute ischaemic stroke. The type of acute ischaemic stroke was classified according to the TOAST classification. We evaluated plasma levels of IL- $1 \alpha$, TNF- $\alpha$, IL-6 and IL-10, E-selectin, P-selectin, sICAM-1 and sVCAM-1, VWF TPA antigen and PAI-1 plasma levels. Neurological deficit score by Scandinavian Stroke Scale (SSS) on admission and disability degree at discharge by Rankin disability scale were obtained for stroke patients.

Results: 120 subjects with acute ischaemic stroke and 123 matched controls (without ischaemic stroke). Patients classified as cardioembolic (CEI) showed, significantly higher median plasma levels of TNF- $\alpha$ IL-6 IL- $1 \alpha$. Furthermore stroke patients classified as lacunar showed, in comparison with other subtypes, significantly lower median plasma levels of TNF- $\alpha$ IL-6, IL-1 $\alpha$. Multiple linear regression performed to investigate about association between some relevant variables and SSS as dependent variable showed a significant correlation between SSS score at admission and diagnostic subtype lacunar or cardioembolic and some inflammatory variable.

Conclusions: Our finding concerning higher plasma levels of immunoinflammation markers in patients with cardioembolic stroke underline the role of inflammation in pathogenesis of cerebral cardioembolism. Infarct size could represent a possible factor influencing immunoinflammatory markers plasma levels and although this issue is not univocally investigated and confirmed it could be conceivable that also infarct site could represent an additional factor that influence immunoinflammatory activation after acute ischaemic stroke. So beyond a possible direct relationship between infarct size and cytokines levels also infarct site could have an additional weight on immunoinflammatory activation of the acute phase. Interestingly in our patients most of lacunar stroke had a subcortical localisation, whereas most of cardioembolic stroke had a cortical localisation. 